\title{
Stereotactic laser ablation for completion corpus callosotomy
}

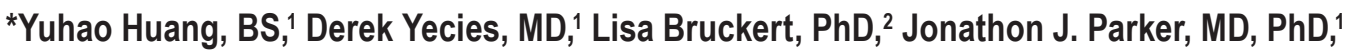 \\ Allen L. Ho, MD, ${ }^{1}$ Lily H. Kim, BA, ${ }^{1}$ Linden Fornoff, MD, ${ }^{1}$ Max Wintermark, MD, MAS, MBA, ${ }^{3}$ \\ Brenda Porter, MD, ${ }^{4}$ Kristen W. Yeom, MD, ${ }^{3}$ Casey H. Halpern, MD, ${ }^{1}$ and Gerald A. Grant, MD ${ }^{1}$
}

Departments of ${ }^{1}$ Neurosurgery, ${ }^{2}$ Pediatrics, ${ }^{3}$ Radiology, and ${ }^{4}$ Neurology, Stanford University School of Medicine, Stanford University, Stanford, California

OBJECTIVE Completion corpus callosotomy can offer further remission from disabling seizures when a prior partial corpus callosotomy has failed and residual callosal tissue is identified on imaging. Traditional microsurgical approaches to section residual fibers carry risks associated with multiple craniotomies and the proximity to the medially oriented motor cortices. Laser interstitial thermal therapy (LITT) represents a minimally invasive approach for the ablation of residual fibers following a prior partial corpus callosotomy. Here, the authors report clinical outcomes of 6 patients undergoing LITT for completion corpus callosotomy and characterize the radiological effects of ablation.

METHODS A retrospective clinical review was performed on a series of 6 patients who underwent LITT completion corpus callosotomy for medically intractable epilepsy at Stanford University Medical Center and Lucile Packard Children's Hospital at Stanford between January 2015 and January 2018. Detailed structural and diffusion-weighted MR images were obtained prior to and at multiple time points after LITT. In 4 patients who underwent diffusion tensor imaging (DTI), streamline tractography was used to reconstruct and evaluate tract projections crossing the anterior (genu and rostrum) and posterior (splenium) parts of the corpus callosum. Multiple diffusion parameters were evaluated at baseline and at each follow-up.

RESULTS Three pediatric (age 8-18 years) and 3 adult patients (age 30-40 years) who underwent completion corpus callosotomy by LITT were identified. Mean length of follow-up postoperatively was 21.2 (range 12-34) months. Two patients had residual splenium, rostrum, and genu of the corpus callosum, while 4 patients had residual splenium only. Postoperative complications included asymptomatic extension of ablation into the left thalamus and transient disconnection syndrome. Ablation of the targeted area was confirmed on immediate postoperative diffusion-weighted MRI in all patients. Engel class I-II outcomes were achieved in 3 adult patients, whereas all 3 pediatric patients had Engel class III-IV outcomes. Tractography in 2 adult and 2 pediatric patients revealed time-dependent reduction of fractional anisotropy after LITT.

CONCLUSIONS LITT is a safe, minimally invasive approach for completion corpus callosotomy. Engel outcomes for completion corpus callosotomy by LITT were similar to reported outcomes of open completion callosotomy, with seizure reduction primarily observed in adult patients. Serial DTI can be used to assess the presence of tract projections over time but does not classify treatment responders or nonresponders.

https://thejns.org/doi/abs/10.3171/2019.5.PEDS19117

KEYWORDS laser interstitial thermal therapy; epilepsy; completion corpus callosotomy; diffusion tensor imaging

I $\mathrm{N}$ patients with medication-resistant epilepsy, neurosurgical options should be explored to control disabling seizures. ${ }^{16}$ Corpus callosotomy can be an effective surgical treatment to minimize injuries resulting from drop attacks and generalized seizures in both adults and children. ${ }^{11,22}$ Partial corpus callosotomy, classically lim- ited to the anterior two-thirds of the callosum, compared to complete corpus callosotomy, which also includes the division of the splenium, have both been utilized to treat this challenging patient population. Partial corpus callosotomy has been associated with a lower risk of permanent disconnection syndrome, whereas complete callosotomy

ABBREVIATIONS $A D=$ axial diffusivity; $A D C=$ apparent diffusion coefficient; $A E D=$ antiepileptic drug; $A F Q=$ Automated Fiber Quantitation; dMRI = diffusion MRI; DTI = diffusion tensor imaging; FA = fractional anisotropy; LGS = Lennox-Gastaut syndrome; LITT = laser interstitial thermal therapy; $M D=$ mean diffusivity; RD = radial diffusivity; $\mathrm{ROI}=$ region of interest; $\mathrm{VNS}=$ vagal nerve stimulator.

SUBMITTED February 27, 2019. ACCEPTED May 3, 2019.

INCLUDE WHEN CITING Published online August 2, 2019; DOI: 10.3171/2019.5.PEDS19117.

${ }^{*}$ Y.H. and D.Y. contributed equally to this work. C.H.H. and G.A.G. share senior authorship of this work. 
may result in better seizure control. ${ }^{14,18,24,25}$ Further, when a prior partial callosotomy has failed to provide meaningful reduction in seizures, completion corpus callosum may be offered to section the residual callosum..$^{6,14}$

Completion surgeries after partial callosotomy have largely been performed through microsurgical approaches, with seizure improvement rates ranging from $40 \%$ to $90 \%{ }^{1,10,14}$ To circumvent morbidities associated with open surgery, radiosurgical completion corpus callosotomy has been attempted. ${ }^{6}$ However, few patients have been treated in this fashion and there remains concern for the longterm risks of radiation exposure, especially in the pediatric population. Laser interstitial thermal therapy (LITT) ablation has emerged as a promising minimally invasive approach to ablate seizure foci. ${ }^{4}$ LITT has been used to target the mesial temporal lobe ${ }^{28}$ hypothalamic hamartomas, ${ }^{29}$ the periventricular region, ${ }^{8}$ and the anterior corpus callosoum. ${ }^{26}$ We have recently reported on the technical aspects of targeting residual corpus callosum with LITT. ${ }^{12}$ In brief, residual corpus callosum is a feasible target for LITT as it is usually a linear target surrounded by the lateral ventricles, which act as excellent heat sinks.

Here we describe our institutional experience using LITT for completion corpus callosotomy. We examined outcomes in a series of 6 patients with intractable epilepsy undergoing LITT completion corpus callosotomy and performed tractography analyses to quantify the ablation effects on interhemispheric crossing fibers of the corpus callosum.

\section{Methods \\ Patient Selection}

After obtaining the approval of our Institutional Review Board, we reviewed the health records of 6 patients who underwent LITT completion corpus callosotomy between January 2015 and January 2018 at Stanford University Medical Center and Lucile Packard Children's Hospital at Stanford. Presurgical evaluation included MRI, video-EEG, and neurological evaluation. As this was a retrospective study, informed consent was not required.

\section{Surgical Procedure}

All surgeries were performed according to the standard techniques of LITT using the Medtronic Visualase system. ${ }^{12,15}$ First, stereotactic CT acquisition via a Medtronic $\mathrm{O}$-arm was completed, followed by placement of the patient's head in Mayfield pins (Integra LifeSciences). Second, after frameless stereotactic registration using the StealthStation Navigation System (Medtronic), a unilateral trajectory was mapped from the right or left parietal region down to the splenium. In 1 patient, a Mazor Robotics Renaissance guidance system was used for trajectory planning and laser guidance (technical details will be published in a separate case report). A twist drill burr hole was made following exposure of the periosteum, and a guide cannula was advanced toward the target. Additional imaging was acquired to confirm optimal placement before transporting the patient to the MRI suite. Finally, in the MRI suite, a test dose was first administered following confirmation of trajectory and target acquisition. High temperature limits were set adjacent to the laser catheter and low temperature limits were set in adjacent critical structures. Real-time MR thermometry was used to monitor heating up to $70^{\circ} \mathrm{C}$ and up to 3 minutes in duration. MRI was obtained to confirm an adequate ablation zone and to rule out hemorrhage.

In 2 patients who required ablation of both the rostrum and splenium of the corpus callosum, we performed LITT entirely in the MRI suite using the ClearPoint platform (MRI Interventions). ${ }^{13}$ Briefly, these patients were transported to the MRI suite for induction of general anesthesia. Next, they were positioned laterally with the right side up in the ClearPoint 4-point fixation head frame. Serial MRI scans were done with planned trajectories to the rostrum and splenium of the corpus callosum. Two ClearPoint MRI-visible SmartGrids were placed along the right frontal region, one for the anterior trajectory toward the splenium and another for the posterior trajectory toward the rostrum. Additional imaging was obtained to confirm optimal grid placements. Next, we mounted the percutaneous SmartFrame, which allows alignment to the planned trajectory. Using an MRI-compatible hand-held twist drill, 3.2-mm burr holes were made. Then, stylets were placed half the distance to the target and additional imaging was performed to ensure a good trajectory. The laser probe was placed down the anterior entry point to reach the splenium. Once the probe was confirmed to be in the correct position on imaging, the target was heated as described above. After the ablation was completed, an additional laser probe was inserted into the posterior entry point to reach the rostrum area, where the ablation procedure was repeated. Ten-millimeter diffusion laser filaments were used for all procedures.

\section{Seizure Outcomes}

Each patient's medical records were reviewed for demographic information, seizure etiology, seizure semiologies, prior neurological surgeries, seizure frequencies, antiepileptic drugs (AEDs) and seizure recurrence. Seizure outcome classification was based on the following modified Engel classification scheme previously used for patients undergoing callosotomy ${ }^{7,14}$ : class I, seizure free from targeted seizure type; class II, major reduction in targeted seizure type; class III, worthwhile reduction in targeted seizure type; and class IV, no worthwhile improvement.

\section{MRI Data Acquisition}

MR images were obtained at $3 \mathrm{~T}$ (GE MR750 Discovery; GE Healthcare) using an 8-channel head coil. Highresolution T1-weighted (3D FSPGR; TE $=3.72 \mathrm{msec}$; TR $=9.23-9.62 \mathrm{msec}$, depending on slice coverage; FOV = $240 \times 240 \mathrm{~mm}^{2}$; acquisition matrix $=256 \times 256$; voxel size $=0.94 \times 0.94 \times 1.00 \mathrm{~mm}^{3}$; orientation $=$ sagittal $), \mathrm{T} 2-$ weighted (FLAIR, TE $=88.9 \mathrm{msec}, \mathrm{TR}=9500 \mathrm{msec}$, FOV $=240 \times 240 \mathrm{~mm}^{2}$, acquisition matrix $=320 \times 256$, voxel size $=0.94 \times 0.94 \times 1.00 \mathrm{~mm}^{3}$, orientation $=$ axial $)$ and diffusion-weighted images were acquired as part of the standard clinical care for surgical treatment and planning. For case 1, diffusion data were acquired with a diffusion tensor-echo planar imaging (DT-EPI) sequence using a 
b value of $1000 \mathrm{~s} / \mathrm{mm}^{2}$ sampling 40 isotropically distributed diffusion directions (dir) (40 dir, $\mathrm{b}=1000, \mathrm{~b} 0=1$, $\mathrm{TE}=80.70 \mathrm{msec}, \mathrm{TR}=15,000 \mathrm{msec}, \mathrm{FOV}=260 \times 260$ $\mathrm{mm}^{2}$, acquisition matrix $=256 \times 256$, voxel size $=1.02 \times$ $1.02 \times 2.50 \mathrm{~mm}^{3}$, number of excitations $[\mathrm{NEX}]=1$ ). For cases 4,5 , and 6 , diffusion data were acquired with a DTEPI sequence $(\mathrm{TE}=60.70 \mathrm{msec}, \mathrm{TR}=8000 \mathrm{msec}$, FOV $=250 \times 250 \mathrm{~mm}^{2}$, acquisition matrix $=256 \times 256$, voxel size $\left.=0.98 \times 0.98 \times 2.00 \mathrm{~mm}^{3}, \mathrm{NEX}=1\right)$ using a $\mathrm{b}$ value of $1000 \mathrm{~s} / \mathrm{mm}^{2}$ sampling 30 isotropically distributed diffusion directions. For all patients, one additional volume was acquired at $b=0$ at the beginning of each scan.

\section{MRI Data Preprocessing}

The open-source software mrDiffusion (https://github. com/LisaBruckert/vistasoft/tree/master/mrDiffusion) implemented in MATLAB R2014a (MathWorks) was used to preprocess the diffusion MRI (dMRI) data. First, we applied a rigid body transformation ${ }^{23}$ to register each diffusion-weighted image to the nondiffusion (b0) image to correct for participants' motion. Second, the b0 image was registered to the participant's T1-weighted image, which had been aligned to the canonical AC-PC (anterior commissure-posterior commissure) orientation. Third, we combined the two transforms obtained from motion correction and alignment to the T1-weighted image and applied it to the raw data. Because nonisotropic voxels may bias the tensor-fitting algorithm and distort both tracking and measurements of diffusion properties, ${ }^{20}$ we resampled the transformed images to $2 \times 2 \times 2-\mathrm{mm}^{3}$ isotropic voxels. Finally, diffusion gradient directions were adjusted to fit the resampled diffusion data ${ }^{17}$ and maps of fractional anisotropy (FA), mean diffusivity (MD), radial diffusivity (RD), and axial diffusivity (AD) were generated using a standard least-squares algorithm.

All dMRI scans were rigorously assessed for head motion. By calculating the magnitude of motion correction in the $x-y-z$ plane of each volume relative to the prior volume, the degree of relative motion was quantified in each participant (in voxels). The number of volumes with a translational relative motion of $\geq 1$ voxel for each dMRI scan was identified. Next, the mean number of volumes with $\geq$ 1 voxel of relative motion across the entire sample was calculated, and the results were as follows: preoperative (pre) mean (SD), 3.25 (6.50); 1 day postoperative (post1), 2.75 (3.40); and $\geq 1$ month postoperative (post 2), 0.33 (0.58). Patients whose pre, post1, or post 2 scan deviated from the calculated mean by more than 2 SDs were excluded from analyses $(\mathrm{n}=0)$. In addition, a Kruskal-Wallis $\mathrm{H}$ test confirmed no significant difference in relative motion among dMRI data collected at the 3 timepoints (chi-square = $0.47, \mathrm{p}=0.82$ ), with a mean rank motion score of 5.75 for pre, 6.75 for post 1 , and 5.33 for post 2 scans.

\section{Diffusion MRI Tractography}

The Automated Fiber Quantitation (AFQ) software package, described in detail by Yeatman et al. (2012), ${ }^{30}$ was used to track and segment 8 subdivisions of the corpus callosum in each patient's native space. In brief, AFQ consists of 3 main processing steps. 1) Whole-brain tractography - a deterministic streamline tracking algorithm ${ }^{19}$-was used to estimate the whole-brain connectome of fiber bundles. The tracking algorithm was seeded with a white matter mask defined as all of the voxels with an FA value above 0.20 . Tracking proceeded in all directions and stopped when FA dropped below 0.15 or when the angle between the last path segment and the next step direction was greater than $30^{\circ}$. 2) Automatic pathway segmentation-fiber bundle segmentation-was performed using a multiple waypoint region of interest (ROI) procedure in which each streamline from the whole-brain connectome was assigned to a specific fiber group if it passed through 2 ROIs that defined the trajectory of the fiber group. ${ }^{27}$ The 8 callosal subdivisions based on the cortical projection zone of the fibers (i.e., occipital, posterior parietal, superior parietal, motor, superior frontal, anterior frontal, orbitofrontal, and temporal projections) were segmented by using 2 ROIs that were placed in homologous regions of each hemisphere, as described in Yeatman et al. ${ }^{30}$ and Dougherty et al. ${ }^{5}$ Because ROIs were defined on a template in Montreal Neurological Institute (MNI) space, a nonlinear transformation ${ }^{9}$ was applied to register these ROIs into each individuals' native space. The central portion of the fiber bundle was defined by clipping each streamline to the portion that spans between the 2 waypoint ROIs and resampling each fiber bundle to 30 equidistant nodes. 3) Automatic pathway refinement and cleaning - the final cleaning of the bundle-was done by computing the robust mean (3D Gaussian covariance of the sample points) and removing fibers that were either more than 4 SDs above the mean fiber length or more than 5 SDs away from the core of the fiber bundle. For each subject, fiber renderings of the corpus callosum were visually inspected to ensure that each subdivision conformed to anatomical norms prior to any statistical analyses.

\section{Results \\ Patient Characteristics}

Between January 2015 and January 2018, 6 patients underwent LITT completion corpus callosotomy at our institution (Table 1). Three patients were adults (age 30-40 years) and 3 patients were children at the time of surgery (age 8-18 years). The adult patients had longer history of seizures (average 28 years, range 22-34 years) than the 3 pediatric patients (average 11 years, range $8-17$ years). Four patients in the cohort had severe developmental delay, including all 3 pediatric patients. All patients had at least 2 seizure types, which included atonic, myoclonic, absence, generalized tonic-clonic (GTC), and focal impaired awareness seizures. Lennox-Gastaut syndrome (LGS) was identified in 4 patients. Structural lesions were identified in 3 patients: one patient was afflicted with Rasmussen encephalitis at the age of 8 years, another had evidence of left hemispheric polymicrogyria, and the third was found to have left frontal dysplasia. Hypothalamic hamartoma was present in one patient but was felt not to be causative of the patient's seizures given it had been previously irradiated and his seizures were not focal or gelastic in nature. 
TABLE 1. Baseline characteristics of study subjects

\begin{tabular}{|c|c|c|c|c|c|c|}
\hline & Pt No. 1 & Pt No. 2 & Pt No. 3 & Pt No. 4 & Pt No. 5 & Pt No. 6 \\
\hline Age at op, yrs & 30 & 30 & 18 & 11 & 8 & 40 \\
\hline Sex & M & $M$ & M & $\mathrm{F}$ & M & M \\
\hline Developmental delay & Severe & Normal & Severe & Severe & Severe & Normal \\
\hline $\begin{array}{l}\text { Duration of epilepsy, } \\
\text { yrs }\end{array}$ & 29 & 22 & 17 & 9 & 8 & 34 \\
\hline Seizure etiology & LGS & Rasmussen encephalitis & LGS, HH & LGS, polymicrogyria & LGS & Frontal dysplasia \\
\hline Seizure types & $\begin{array}{l}\text { Atonic, absence, } \\
\text { tonic-clonic }\end{array}$ & $\begin{array}{l}\text { GTC, focal impaired } \\
\text { awareness }\end{array}$ & $\begin{array}{l}\text { GTC, myoclonic, } \\
\text { atonic }\end{array}$ & $\begin{array}{l}\text { GTC, atonic, focal } \\
\text { impaired awareness }\end{array}$ & $\begin{array}{l}\text { Atonic, head } \\
\text { drop }\end{array}$ & $\begin{array}{l}\text { Atonic, focal impaired } \\
\text { awareness }\end{array}$ \\
\hline Prior op & $\mathrm{AC}$ & $\begin{array}{l}\text { Lt hemispherectomy w/ } \\
\text { splenial \& occipital } \\
\text { remnant }\end{array}$ & $\begin{array}{l}\mathrm{PC}, \text { CyberKnife } \\
\text { radiosurgery }\end{array}$ & $\mathrm{AC}, \mathrm{VNS}$ & $P C$ & $\begin{array}{l}\text { Lt frontotemporal resec- } \\
\quad \text { tion, AC, \& VNS }\end{array}$ \\
\hline
\end{tabular}

$\mathrm{AC}=$ anterior callosotomy $; \mathrm{HH}=$ hypothalamic hamartoma; $\mathrm{PC}=$ partial callosotomy; $\mathrm{pt}=$ patient.

\section{Prior Operations}

Three patients had previously received microsurgical anterior corpus callosotomy (Table 1). Patients 3 and 5 had undergone a partial corpus callosotomy, which left intact the rostrum/genu and splenium of the corpus callosum. Patient 2 underwent a left hemispherectomy with occipital and splenial remnants. Further, CyberKnife radiosurgery was performed in 1 patient for treatment of his hypothalamic hamartoma. A vagal nerve stimulator (VNS) was previously implanted in 2 patients.

\section{Surgical Characteristics and Complications}

All patients underwent ablation of the splenium (Table 2). Additional ablation at the rostrum was performed in 2 patients. Exemplar trajectories targeting the rostrum/genu and splenium of the corpus callosum are shown for patient 5 (Fig. 1A). In patient 2, who underwent a left hemispherectomy, an occipital remnant was also ablated in addition to the splenium. There were 3 perioperative complications, none of which resulted in long-term negative effects. One patient without developmental delay (patient 6) experienced a transient disconnection syndrome characterized by right-hemifield neglect, lack of right-sided stereognosis, and apathy, which resolved over the course of 6 months. Patient 3 had transient bradycardia in response to gadolinium injection on postoperative MR imaging. Patient 5 had MRI evidence of extension of ablation into the left postero-medial thalamus without obvious neurological symptoms (Fig. 1C). There was no clinical neurologi- cal deficit secondary to this heating event, and the FLAIR signal had resolved by 3.5 months postoperatively (Fig. 1D) with no indication of encephalomalcia on structural MRI (in contrast to the region of intended splenial ablation). This unintended heat spread was first noticed during the initial burn with an energy of $9.75 \mathrm{~W}(65 \%$ laser power), which is a standard power setting for our institution and others. No heat spread into the thalamic region was observed during the preceding test dose. Several seconds into the first ablation a "frond" of heating was noted extending into the posterior thalamic region (Fig. 1E). The heat appeared to be spreading from the diffusing tip of the laser catheter. The ablation was stopped, the laser catheter was withdrawn, a new test dose was given, and a low temperature was selected for safety in the region of prior heating (Fig. 1F). We hypothesized that the laser filament may have been "hubbed" to the end of the laser catheter, which may have limited effective laser cooling via circulating irrigation, leading to more robust heat projection than anticipated. The authors again emphasize the importance of vigilance to thermometry at all times during the procedure and cognizance of the several-second time delay that exists between changes to laser parameters and updates to the temperature map the surgeon is able to monitor.

\section{Seizure Outcomes}

The mean follow-up duration was 21.2 months (range 12-34 months). Durable reduction in the targeted seizure type was achieved in the 3 adult patients, with 2 patients achieving Engel class I outcomes (Table 3). In contrast,

TABLE 2. Surgical procedure and complications

\begin{tabular}{lcccccc}
\hline & Pt No. 1 & Pt No. 2 & Pt No. 3 & Pt No. 4 & Pt No. 5 & Pt No.6 \\
\hline Ablation target & Splenium & $\begin{array}{c}\text { Splenium \& occipital } \\
\text { remnant }\end{array}$ & Rostrum \& splenium & Splenium & Rostrum \& splenium & Splenium \\
\hline Perioperative complications & None & None & $\begin{array}{c}\text { Transient bradycardia } \\
\text { \& bigeminy }\end{array}$ & None & $\begin{array}{c}\text { Asymptomatic extension } \\
\text { of ablation into posterior } \\
\text { thalamus }\end{array}$ & $\begin{array}{c}\text { Disconnection } \\
\text { syndrome }\end{array}$ \\
\hline Length of hospitalization (days) & 1 & 1 & 8 & 3 & 3 \\
\hline
\end{tabular}


pediatric patients experienced either a transient improvement or no change at all in seizure frequency. Pediatric patients 3 and 5 experienced temporary reduction in the targeted seizure type for 1 and 4 months, respectively; however, they subsequently began to have seizures again with increasing frequency. The other pediatric patient (patient 4) continued to experience atonic seizures after the completion surgery. This patient went on to receive microsurgical sectioning of the splenium 2 months after the laser completion corpus callosotomy. There was no meaningful reduction of her atonic seizures despite this additional intervention. For all patients, neither the number nor dose of AEDs changed substantially before and after surgery.

\section{Imaging Outcomes}

On postoperative day 1 , expected areas of thermal ablation were identified on diffusion-weighted imaging (Fig. 2). These changes were attenuated at the next follow-up scan 1 or more months later. Tractography of the corpus callosum using AFQ was successfully completed in 4 of 6 patients. We could not perform tractography in 2 patients due to either heavily distorted anatomy (patient 2) or incompatibility of scanning parameters between the pre- and postoperative diffusion tensor imaging (DTI) (patient 3). Preoperatively, the splenium fiber projections were reconstructed in all 4 patients, with 1 patient (patient 5) also showing fiber projections in the genu and rostrum area (Fig. 3). On postoperative day 1, despite evident ablation on diffusion-weighted imaging (Fig. 2), interhemispheric fibers of the corpus callosum could still be tracked in 3 patients, although at much lower density. At the next follow-up (1 month or more later), substantially fewer fiber projections could be reconstructed using tractography (Fig. 3). Note that for patient 1, crossing fiber projections were not present on postoperative day 1 , but some crossing fiber projections were reconstructed at the next scan date. Quantitatively, a marked decrease in FA of the corpus callosum was observed 1 day postsurgery, while the apparent diffusion coefficient (ADC) was nearly normal (Fig. 4). At greater than 1 month postsurgery, we observed a further decrease in FA, while ADC showed variable changes ( 2 increased while 1 decreased). Of note, as shown in Table 3, patients 1 and 6 achieved Engel I outcomes while patients 4 and 5 showed Engel III-IV outcomes. We did not observe any substantial difference in the decrease in FA or ADC that are stratified by these outcomes (Fig. 4).

\section{Discussion}

We present our initial experience with LITT completion corpus callosotomy in 6 patients and report clinical and imaging outcomes over time. Overall, LITT completion corpus callosotomy was performed with a reasonable safety profile and minimal complications. One patient had an extension of the splenial ablation into the posteromedial left thalamus, but the patient remained asymptomatic through the hospitalization and at subsequent follow-up visits. Disconnection syndrome occurred in one adult patient, which was characterized by right hemifield neglect
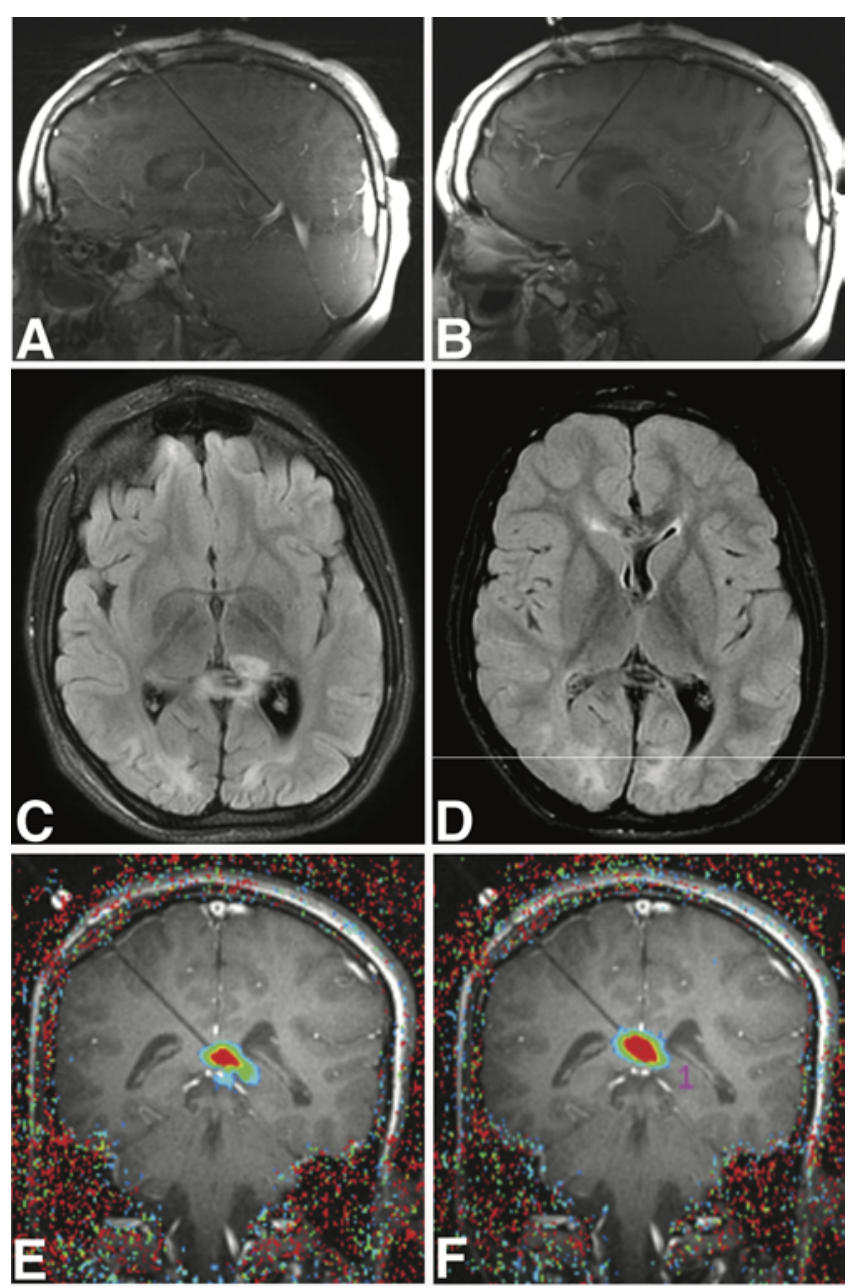

FIG. 1. Representative trajectories for rostral/genu and splenial targets (A and $B$ ). A: T1-weighted imaging showing probe trajectory reaching the splenium of the corpus callosum. B: T1-weighted imaging showing probe trajectory reaching the rostrum/genu of the corpus callosum. Extension of laser ablation into the left postero-medial thalamus ( $\mathrm{C}$ and D). C: T2-weighted FLAIR imaging showing evidence of ablation and small extension into the left thalamus. D: T2-weighted FLAIR imaging 3.5 months postoperatively showing resolution of the prior FLAIR signal in the thalamus. Real-time ablation dynamics (E and F). E: Temperature dynamic during the first burn indicating extension into the posteriorthalamic region. The ablation was immediately stopped and the laser catheter was pulled back. F: Temperature dynamic during the second burn indicating the posterior-thalamic region was no longer at risk. Figure is available in color online only.

and a lack of right-sided stereognosis. With rehabilitation, this patient's symptoms largely resolved after 6 months. In contrast, traditional microsurgical corpus callosotomy carries substantial surgical risks. Complications associated with an open craniotomy and vessel dissection include meningitis, subdural hematoma, venous or arterial stroke, subgaleal fluid collection, and cerebral hemorrhage. Hemiparesis and speech delay have also been reported after callosotomy with microsurgical dissection.,14 Compared to stereotactic radiosurgery, LITT obviates the long-term radiation risks as well as postradiational complications such as radiation necrosis and cerebral edema. 
Huang et al.

TABLE 3. Seizure outcomes following laser ablation

\begin{tabular}{|c|c|c|c|c|c|c|}
\hline & Pt No. 1 & Pt No. 2 & Pt No. 3 & Pt No. 4 & Pt No. 5 & Pt No. 6 \\
\hline Engel outcome class & I & II & IV & IV & III & I \\
\hline FU, mos & 32 & 34 & 18 & 16 & 15 & 12 \\
\hline Targeted Sz type & Atonic & GTC & GTC & Atonic & Atonic & Atonic \\
\hline $\begin{array}{l}\text { Recurrence of targeted } \\
\text { Sz type, mos }\end{array}$ & - & 12 & 1 & $<1$ & 4 & - \\
\hline $\begin{array}{l}\text { Frequency of targeted } \\
\text { Szs before op }\end{array}$ & 2/day & $1 / w k$ & 10-15/day & Several/day & 10-15/day & $3-4 /$ wk \\
\hline $\begin{array}{l}\text { Frequency of targeted } \\
\text { Szs at last FU }\end{array}$ & None & $1-2 / y r$ & 10-15/day & Several/day & 5-10/day & None \\
\hline Additional ops & - & - & - & $\begin{array}{l}\text { Microsurgical comple- } \\
\text { tion }\end{array}$ & - & - \\
\hline \multicolumn{7}{|l|}{ AEDs* } \\
\hline Prior to op & CLZ, PHB, LEV, RFM & CLZ, CAR, LAC & CLZ, LAM, LEV & CLZ, CLB, RFM & CLZ, LAC, VPA & $\begin{array}{l}\text { CLB, PHT, RFM, } \\
\text { ESL }\end{array}$ \\
\hline Last clinic visit & $\begin{array}{l}\text { CLZ (-), PHT, LEV, } \\
\text { VPA }\end{array}$ & CAR, LAC (+) & CLZ, LAM (+), LEV & CLZ, CLB (+), RFM (+) & LAC $(+)$ & $\begin{array}{l}\text { CLB, PHT, RFM, } \\
\text { ESL (no change) }\end{array}$ \\
\hline
\end{tabular}

$\mathrm{CAR}$ = carbamazepine; $\mathrm{CLB}=$ clobazam; $\mathrm{CLZ}$ = clonazepam; ESL = eslicarbazepine; FU = follow-up; $\mathrm{LAC}$ = lacosamide; $\mathrm{LAM}$ = lamotrigine; $\mathrm{LEV} \mathrm{=} \mathrm{levetiracetam;} \mathrm{PHB}$ = phenobarbital; $\mathrm{PHT}=$ phenytoin; $\mathrm{RFM}=$ rufinamide; $\mathrm{Sz}=$ seizure VPA = valproic acid.

${ }^{*}(+)$ indicates higher dose whereas $(-)$ indicates lower dose at last clinic visit compared with prior to surgery.

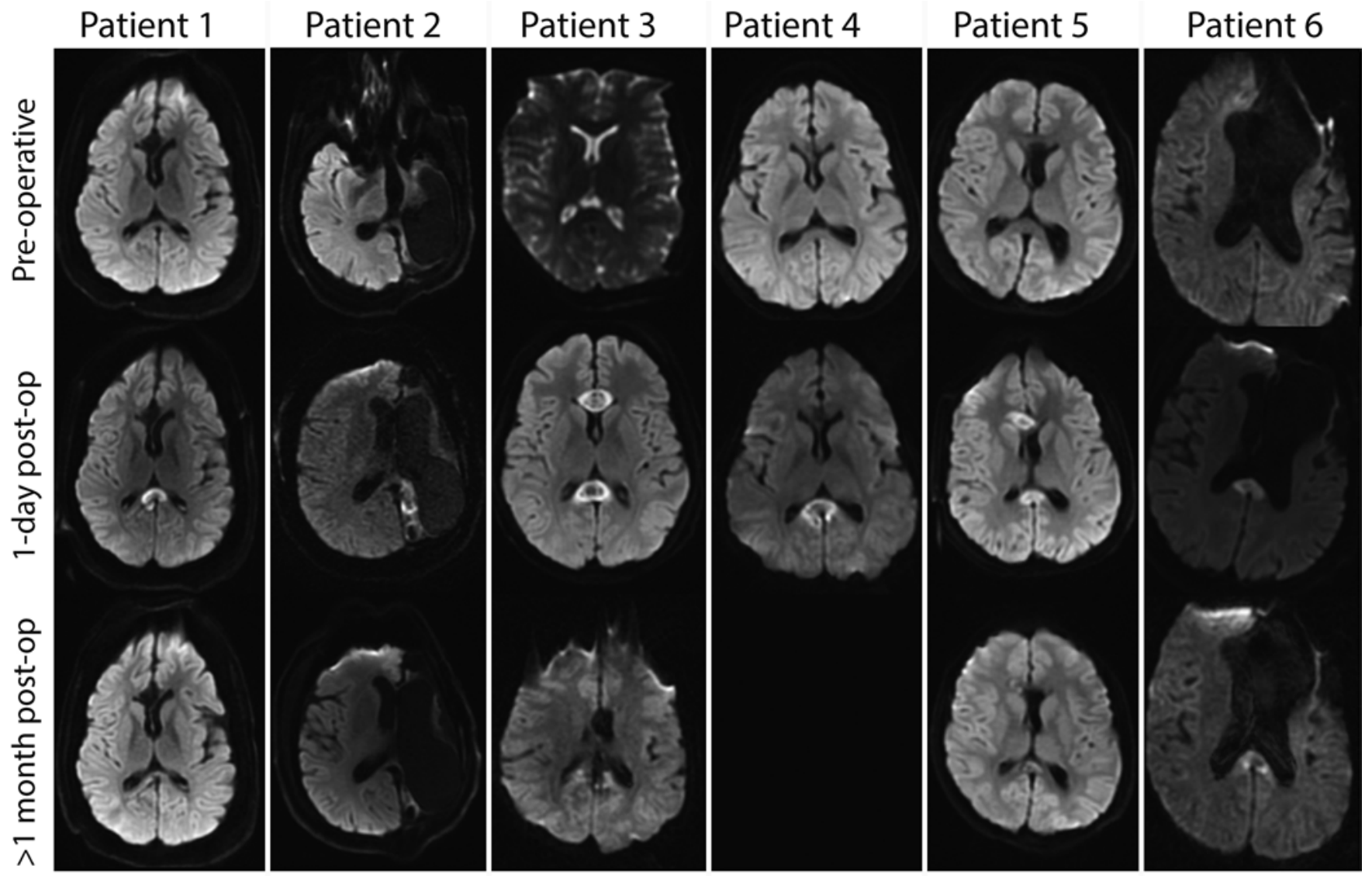

FIG. 2. Time-dependent effect of laser ablation. One day postsurgery, laser ablation changes are present on diffusion-weighted imaging in the expected splenial and/or rostral/genu corpus callosum. One month or longer postsurgery, previously seen abnormal signal associated with laser ablation is attenuated. 

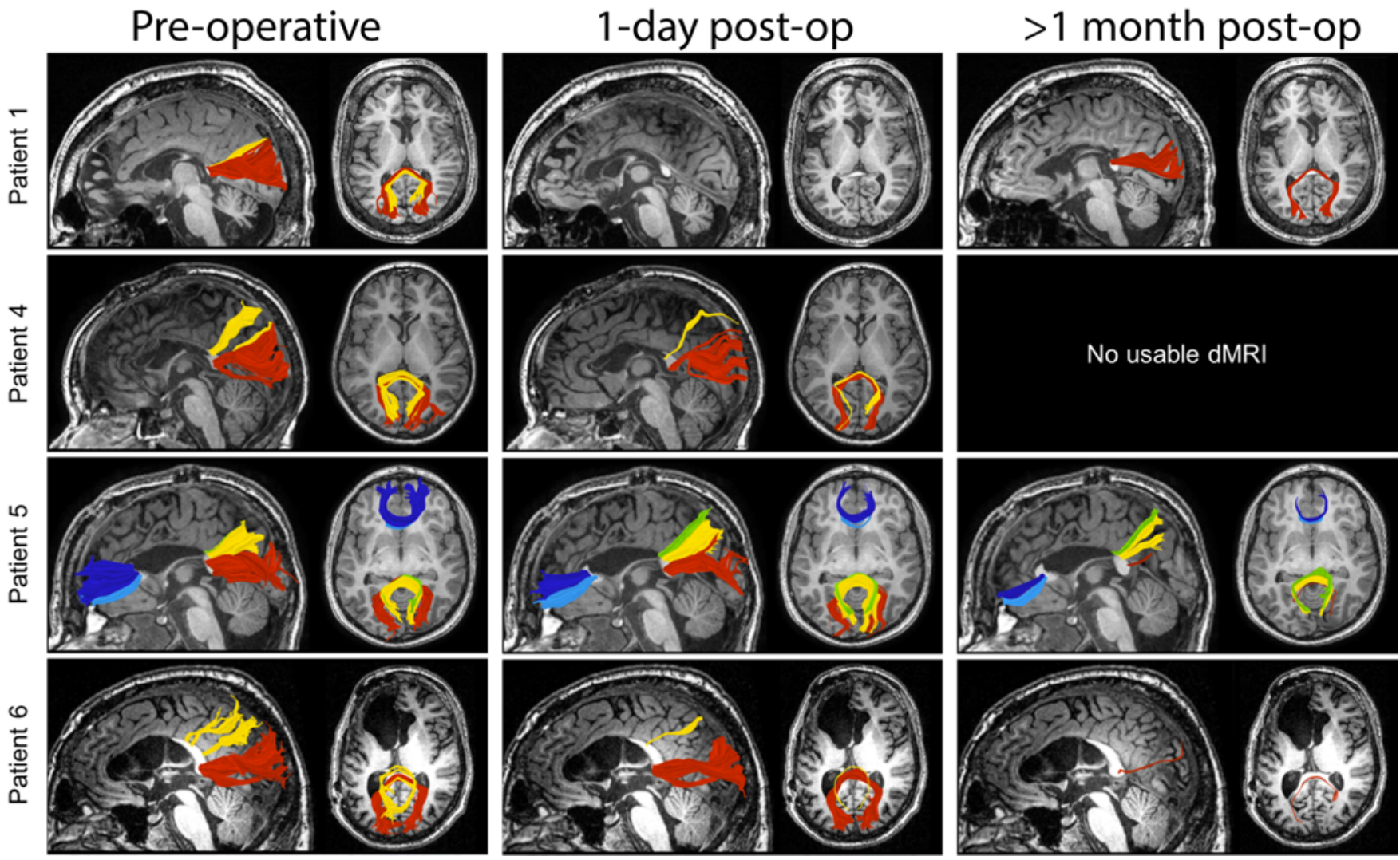

FIG. 3. Effect of laser ablation on DTI fiber tracking. Tractography shows robust crossing fiber projections in the splenium and/or the rostrum of the corpus callosum prior to surgery. One day postsurgery, residual crossing fiber projections were seen, although more attenuated in appearance compared to MRI prior to surgery. In patient 1 fiber projections could not be performed on postoperative day 1. After 1 month or more, more attenuated appearance of the fiber projections was observed for patients 5 and 6 . Patient 1 demonstrated some residual fiber projections. Patient 4 did not have additional DTI data after the first scan on postoperative day 1 . Figure is available in color online only.

The efficacy of LITT is comparable to that demonstrated in other published series of completion corpus callosotomy. In an earlier study by Fuiks et al., open completion surgeries resulted in improvement in 5 out of 10 patients, with response to the initial partial corpus callosotomy being predictive of outcome following completion..$^{10}$ Jalilian et al. reported outcomes of 7 patients who underwent completion surgeries (6 microsurgical dissection, 1 stereotactic radiosurgery). ${ }^{14}$ In this cohort, 3 patients achieved Engel class I-II outcomes, 1 patient achieved Engel class III outcome, and 3 patients achieved Engel class IV outcomes. Bower et al. reported that 4 out of 5 patients had favorable outcomes in reducing drop attacks, defined as Engel classes I-III. ${ }^{1}$ Finally, a recent case series by Palma et al. showed that LITT completion corpus callosotomy in 2 patients resulted in good seizure control. ${ }^{21}$ In our institutional series, we found similar efficacy, with $2 / 6$ patients obtaining Engel I outcomes, 1/6 Engel II, 1/6 Engel III, and 2/6 Engel IV (Table 3). Additionally, we noted that LITT appears to have been of greater benefit to our adult patients than to our pediatric patients. There are several differences between the pediatric and adult patients in this series that may in part explain this discrepancy. The pediatric patients in this series all had severe developmental delay, while 2 of the adult patients had relatively normal cognition. The pediatric patients also had worse preoperative seizure burdens than the adult patients, suggesting both that they had more severe epilepsy and that they had had less efficacy from their initial disconnection procedure, which prior literature suggests portends a lower likelihood of success following completion. As up to $50 \%$ of patients from open surgical series derive no benefit from completion corpus callosotomy, the treatment failures in our pediatric patients were unlikely to have been due to the inadequate disconnection from LITT, but were likely to have been due to the limited efficacy of completion procedures. Some have advocated for a more extensive disconnection between the two hemispheres to include the anterior and posterior commissures, which are a theoretical persistent connection pathway by which seizures may continue to spread. ${ }^{2}$ Further, in the single patient (patient 4) who went on to have a microsurgical revision completion corpus callosotomy after LITT, the revision operation did not confer any additional seizure benefit. This result suggests that the initial LITT technique did not yield poor seizure outcomes due to a failure to adequately disconnect, but rather due to the modest success rate of completion corpus callosotomies overall. 

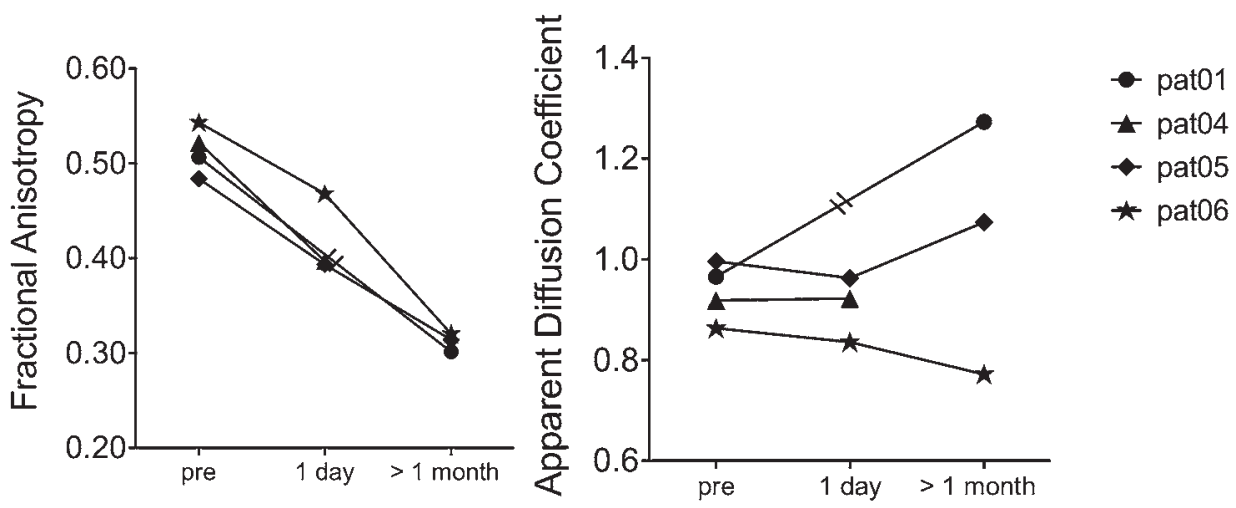

FIG. 4. Diffusion parameters in the transected corpus callosum. Left: FA shows marked decrease 1 day postsurgery and further decrease 1 month or more after surgery. Patient 4 (pat04) did not have additional DTI data after the first scan on postoperative day 1. Right: ADC remains nearly normal 1 day postsurgery and appears to diverge 1 month or more after surgery. Two patients showed increases in ADC while one patient showed a small decrease in ADC. pre = preoperative.

On imaging analysis, all patients showed clear evidence of laser ablation on diffusion-weighted imaging. In 4 patients, we were able to successfully reconstruct the residual crossing fiber projections in the splenium and/or the genu/rostrum on preoperative DTI. Immediately following LITT, we noted persistence of these tracts, though there was marked reduction in fiber projection density over time. These results suggest there might be residual directionality to the thermally ablated axons shortly after surgery, which diminishes over time. In contrast to the gradual effect of LITT, some have argued that the effect of microsurgical disconnection on DTI fiber projections is largely immediate. ${ }^{3}$ This is important as the effectiveness of disconnection may not be readily apparent on DTI when compared to microsurgical sectioning of the fiber tracts. Finally, we quantified FA of the corpus callosum and showed a steady decline over time. The FA decline was similar for the 4 patients with DTI data, irrespective of their treatment outcomes. This suggests that DTI fiber projections may not be sensitive enough or of the correct modality to be able to delineate responders or nonresponders to completion corpus callosotomy. Future multiinstitutional and longitudinal study will be required to confirm this finding.

\section{Study Limitations}

There are several limitations to this study. First, the majority of patients in our cohort received their initial corpus callosotomy procedure at an outside institution. As such, we do not have access to medical records surrounding their initial procedure, including length of stay and complications, which may have affected their seizure outcomes. Second, our cohort size is small, and thus future multicenter study is required to further characterize seizure outcomes and DTI findings. Additionally, given our follow-up duration of at most 3 years, our study can only serve to inform short-term seizure outcomes for these patients. Similarly, DTI imaging could not be obtained on long-term follow-up. As such, our DTI results primarily characterize short-term effects of laser ablation $(<6$ months).

\section{Conclusions}

LITT is a safe, minimally invasive approach for completion corpus callosotomy. Engel outcomes were similar to reported outcomes of open completion callosotomy, with seizure reduction primarily observed in adult patients. Serial DTI can be used to assess the presence of tract projections over time but does not classify treatment responders or nonresponders.

\section{References}

1. Bower RS, Wirrell E, Nwojo M, Wetjen NM, Marsh WR, Meyer FB: Seizure outcomes after corpus callosotomy for drop attacks. Neurosurgery 73:993-1000, 2013

2. Chandra SP, Kurwale NS, Chibber SS, Banerji J, Dwivedi R, Garg A, et al: Endoscopic-assisted (through a mini craniotomy) corpus callosotomy combined with anterior, hippocampal, and posterior commissurotomy in Lennox-Gastaut syndrome: a pilot study to establish its safety and efficacy. Neurosurgery 78:743-751, 2016

3. Choudhri AF, Whitehead MT, McGregor AL, Einhaus SL, Boop FA, Wheless JW: Diffusion tensor imaging to evaluate commissural disconnection after corpus callosotomy. Neuroradiology 55:1397-1403, 2013

4. Curry DJ, Gowda A, McNichols RJ, Wilfong AA: MR-guided stereotactic laser ablation of epileptogenic foci in children. Epilepsy Behav 24:408-414, 2012

5. Dougherty RF, Ben-Shachar M, Deutsch GK, Hernandez A, Fox GR, Wandell BA: Temporal-callosal pathway diffusivity predicts phonological skills in children. Proc Natl Acad Sci U S A 104:8556-8561, 2007

6. Eder HG, Feichtinger M, Pieper T, Kurschel S, Schroettner O: Gamma knife radiosurgery for callosotomy in children with drug-resistant epilepsy. Childs Nerv Syst 22:1012-1017, 2006

7. Engel J (ed): Surgical Treatment of the Epilepsies, ed 2. New York: Raven Press, 1993

8. Esquenazi Y, Kalamangalam GP, Slater JD, Knowlton RC, Friedman E, Morris SA, et al: Stereotactic laser ablation of epileptogenic periventricular nodular heterotopia. Epilepsy Res 108:547-554, 2014

9. Friston KJ, Ashburner J: Generative and recognition models for neuroanatomy. Neuroimage 23:21-24, 2004

10. Fuiks KS, Wyler AR, Hermann BP, Somes G: Seizure outcome from anterior and complete corpus callosotomy. J Neurosurg 74:573-578, 1991 
11. Graham D, Tisdall MM, Gill D: Corpus callosotomy outcomes in pediatric patients: a systematic review. Epilepsia 57:1053-1068, 2016

12. Ho AL, Miller KJ, Cartmell S, Inoyama K, Fisher RS, Halpern CH: Stereotactic laser ablation of the splenium for intractable epilepsy. Epilepsy Behav Case Rep 5:23-26, 2016

13. Ho AL, Sussman ES, Pendharkar AV, Le S, Mantovani A, Keebaugh AC, et al: Improved operative efficiency using a real-time MRI-guided stereotactic platform for laser amygdalohippocampotomy. J Neurosurg 128:1165-1172, 2018

14. Jalilian L, Limbrick DD, Steger-May K, Johnston J, Powers AK, Smyth MD: Complete versus anterior two-thirds corpus callosotomy in children: analysis of outcome. J Neurosurg Pediatr 6:257-266, 2010

15. Jethwa PR, Barrese JC, Gowda A, Shetty A, Danish SF: Magnetic resonance thermometry-guided laser-induced thermal therapy for intracranial neoplasms: initial experience. Neurosurgery 71 (1 Suppl Operative):133-145, 2012

16. Kwan P, Brodie MJ: Early identification of refractory epilepsy. N Engl J Med 342:314-319, 2000

17. Leemans A, Jones DK: The $B$-matrix must be rotated when correcting for subject motion in DTI data. Magn Reson Med 61:1336-1349, 2009

18. Mamelak AN, Barbaro NM, Walker JA, Laxer KD: Corpus callosotomy: a quantitative study of the extent of resection, seizure control, and neuropsychological outcome. J Neurosurg 79:688-695, 1993

19. Mori S, Crain BJ, Chacko VP, van Zijl PC: Three-dimensional tracking of axonal projections in the brain by magnetic resonance imaging. Ann Neurol 45:265-269, 1999

20. Oouchi H, Yamada K, Sakai K, Kizu O, Kubota T, Ito H, et al: Diffusion anisotropy measurement of brain white matter is affected by voxel size: underestimation occurs in areas with crossing fibers. AJNR Am J Neuroradiol 28:11021106, 2007

21. Palma AE, Wicks RT, Popli G, Couture DE: Corpus callosotomy via laser interstitial thermal therapy: a case series. J Neurosurg Pediatr 23:303-307, 2018

22. Park MS, Nakagawa E, Schoenberg MR, Benbadis SR, Vale FL: Outcome of corpus callosotomy in adults. Epilepsy Behav 28:181-184, 2013

23. Rohde GK, Barnett AS, Basser PJ, Marenco S, Pierpaoli C: Comprehensive approach for correction of motion and distortion in diffusion-weighted MRI. Magn Reson Med 51:103114,2004

24. Shim KW, Lee YM, Kim HD, Lee JS, Choi JU, Kim DS: Changing the paradigm of 1-stage total callosotomy for the treatment of pediatric generalized epilepsy. J Neurosurg Pediatr 2:29-36, 2008
25. Spencer SS, Spencer DD, Sass K, Westerveld M, Katz A Mattson R: Anterior, total, and two-stage corpus callosum section: differential and incremental seizure responses. Epilepsia 34:561-567, 1993

26. Tao JX, Issa NP, Wu S, Rose S, Collins J, Warnke PC: Interstitial stereotactic laser anterior corpus callosotomy: a report of 2 cases with operative technique and effectiveness. Neurosurgery [epub ahead of print], 2018

27. Wakana S, Caprihan A, Panzenboeck MM, Fallon JH, Perry M, Gollub RL, et al: Reproducibility of quantitative tractography methods applied to cerebral white matter. Neuroimage 36:630-644, 2007

28. Wicks RT, Jermakowicz WJ, Jagid JR, Couture DE, Willie JT, Laxton AW, et al: Laser interstitial thermal therapy for mesial temporal lobe epilepsy. Neurosurgery 79 (Suppl 1):S83-S91, 2016

29. Xu DS, Chen T, Hlubek RJ, Bristol RE, Smith KA, Ponce FA, et al: Magnetic resonance imaging-guided laser interstitial thermal therapy for the treatment of hypothalamic hamartomas: a retrospective review. Neurosurgery 83:1183-1192, 2018

30. Yeatman JD, Dougherty RF, Myall NJ, Wandell BA, Feldman HM: Tract profiles of white matter properties: automating fiber-tract quantification. PLoS One 7:e49790, 2012

\section{Disclosures}

The authors report no conflict of interest concerning the materials or methods used in this study or the findings specified in this paper.

\section{Author Contributions}

Conception and design: Grant, Yecies, Ho. Acquisition of data: Huang, Yecies, Ho, Kim. Analysis and interpretation of data: Huang, Yecies, Bruckert. Drafting the article: Huang, Bruckert. Critically revising the article: Grant, Huang, Yecies, Bruckert, Parker, Fornoff, Wintermark, Porter, Yeom, Halpern. Reviewed submitted version of manuscript: Grant, Huang, Yecies. Study supervision: Grant.

\section{Correspondence}

Gerald A. Grant: Stanford University School of Medicine, Stanford,CA.ggrant2@stanford.edu. 